\title{
A Large Mastoid Cholesterol Granuloma Eroding the Dura and Sigmoid Sinus
}

Hui-Shan Hsieh ${ }^{1}$, Ming-Ying Zhuo ${ }^{1}$, Ta-Jen Lee ${ }^{1}$, Xiao-Hui Zhemg ${ }^{2}$ and Chung-Feng Hwang ${ }^{3 *}$

${ }^{1}$ Department of Otolaryngology, Chang Gung Memorial Hospital, Xiamen Medical Center, China

${ }^{2}$ Department of Pathology, Chang Gung Memorial Hospital, Xiamen Medical Center, China

${ }^{3}$ Department of Otolaryngology, Kaohsiung Chang Gung Memorial Hospital and Chang Gung University College of Medicine, Kaohsiung, Taiwan (R.O.C.)

\begin{abstract}
Introduction: Large mastoid cholesterol granulomas, particularly aggressive ones, are rare. We present the first case of a mastoid cholesterol granuloma with a nonspecific clinical presentation, and aggressive behavior, destroying the petrous internal cortical plate, dura mater, and sigmoid sinus wall. Radiographic and histological examinations can help to distinguish such granulomas from others.

Case presentation: A 50-year-old male presented with left otalgia, left blood-stained otorrhea, and a left temporal headache radiating to the mastoid. The radiographic examination revealed a left-sided, soft-tissue mass extending into the posterior fossa and compressing the sigmoid sinus inward. The histopathological features were consistent with a cholesterol granuloma. He underwent a cortical mastoidectomy to explore the lesion and macroscopic complete resection was achieved.
\end{abstract}

Conclusion: This is the first case of a mastoid cholesterol granuloma eroding the dura and sigmoid sinus. This case illustrates the need to consider cholesterol granuloma in daily clinical practice. Such lesions can exhibit non-characteristic features and have an aggressive nature.

Keywords: Cholesterol granuloma; Mastoid; Aggressive; Dura; Sigmoid sinus

\section{Introduction}

A cholesterol granuloma is a foreign body giant cell formation in response to cholesterol crystals. Cholesterol granulomas are uncommon in the aerated bones of the skull, and are most frequently found in the petrous apex, as a result of inflammatory reactions in obstructed air spaces [1]. The decreased pressure results in hemorrhage, with the hemoglobin catabolized into cholesterol, which is relatively resistant to absorption by giant cells; this process results in a granulomatous reaction [2]. They can manifest as slowly growing aggressive lesions that cause bone erosion.

Mastoid cholesterol granulomas are often accompanied by middleear lesions, such as chronic otitis media, adhesive otitis media, and middle-ear cholesteatoma. Contrasting petrous apex cholesterol granulomas, mastoid cholesterol granulomas tend to be benign and are usually nonaggressive and nonprogressive [3]. Recently, we treated a huge, aggressive mastoid cholesterol granuloma; this the first reported case of such a lesion not only eroding into the cranial cavity but also the sigmoid sinus.

\section{Case Presentation}

A 50-year-old male had had bilateral intermittent purulent otorrhea since childhood. In the past month, he developed left otalgia, left bloodstained otorrhea, and left temporal headache radiating to the mastoid. $\mathrm{He}$ had suffered from bilateral hearing gradual deterioration over decades, particularly on the left. There were no associated symptoms of vertigo, imbalance, or tinnitus.

The physical examination revealed a left tympanic membrane perforation and the rest of the examination was unremarkable. Puretone audiometry revealed a left moderate mixed hearing loss with mild sensorineural hearing loss in the opposite ear.

Computed tomography (CT) revealed a large soft-tissue mass in

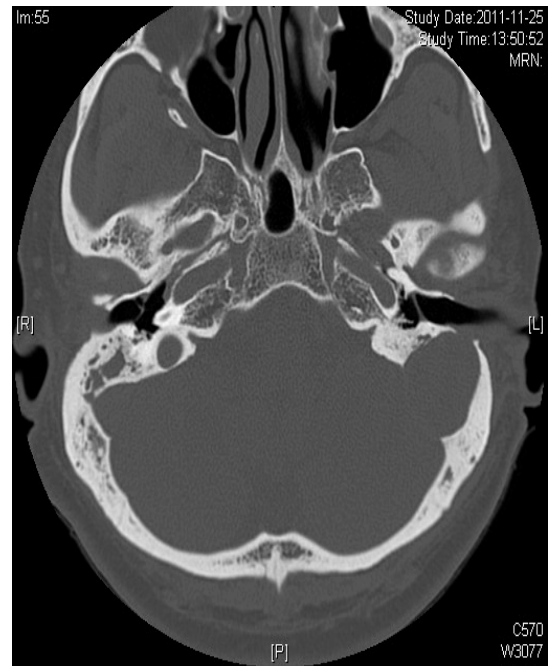

Figure 1: Computed tomography (CT) revealed a large soft-tissue mass in the left temporal region protruding to the posterior fossa with bone erosion.

*Corresponding author: Chung-Feng Hwang, Department of Otolaryngology, Kaohsiung Chang Gung Memorial Hospital and Chang Gung University College of Medicine, 123 Ta-Pei Road, Niao-Sung District, Kaohsiung 833, Taiwan (R.O.C.), Tel: +886-7-7317123 ext.2533; Fax: +886-7-7313855; E-mail: cfhwang@hotmail.com

Received February 28, 2014; Accepted March 22, 2014; Published March 24 2014

Citation: Hsieh HS, Zhuo MY, Lee TJ, Zhemg XH, Hwang CF (2014) A Large Mastoid Cholesterol Granuloma Eroding the Dura and Sigmoid Sinus. J Cytol Histol 5: 236. doi:10.4172/2157-7099.1000236

Copyright: @ 2014 Hsieh HS, et al. This is an open-access article distributed under the terms of the Creative Commons Attribution License, which permits unrestricted use, distribution, and reproduction in any medium, provided the original author and source are credited. 
the left temporal region protruding to the posterior fossa with bone erosion (Figure 1). Magnetic resonance imaging (MRI) showed the soft-tissue mass extending toward the posterior fossa and compressing the sigmoid sinus inward with high-signal intensity on $\mathrm{T} 1$ and T2weighted images (Figure 2). A provisional diagnosis of a left mastoid cholesterol granuloma secondary to cholesteatoma was made.

The patient underwent an extended cortical mastoidectomy via a postauricular approach. The operation revealed large bone deficits in the posterior wall of the canal, cerebellum, and sigmoid sinus. A sharp dissector was used to separate the cystic wall from the dura and sigmoid sinus carefully (Figure 3 ). As the tumor adhered strongly to and had eroded the dura and sigmoid sinus, there was cerebrospinal fluid (CSF) leakage and a mild sigmoid sinus eruption during the procedure. These deficits were sealed with SURGICEL and Gelfoam supplemented with fat. The ossicular chain was intact and no cholesteatoma was seen, although there was some granulation in the epitympanum and mesotympanum. The histopathological examination revealed an irregularly shaped mass surrounded by histiocytes and multinucleated giant cells. Fresh hemorrhage and hemosiderin pigment were apparent (Figure 4). At the 6-month follow-up, there was improvement in the left headache and giddiness. The patient was well at the 12-month follow-up, but his hearing remained at the same level.

\section{Discussion}

Histologically, a cholesterol granuloma is a foreign body giant cell formation responding to cholesterol crystals. The pathogenesis of cholesterol granuloma is uncertain and two theories have been

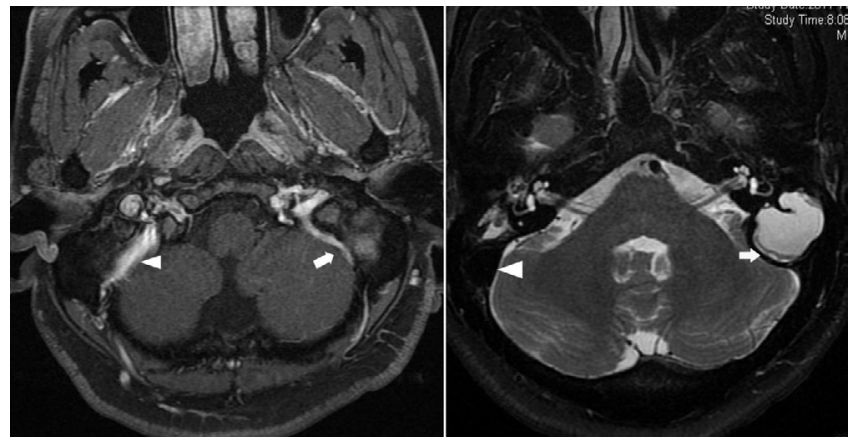

Figure 2: T1- (left) and T2- (right) weighed axial MRI showed a well-defined high-signal-intensity, soft-tissue mass protruding to the left posterior fossa. The left sigmoid sinus (arrow) was compressed by the cholesterol granuloma, compared with the normal right sigmoid sinus (arrowhead).

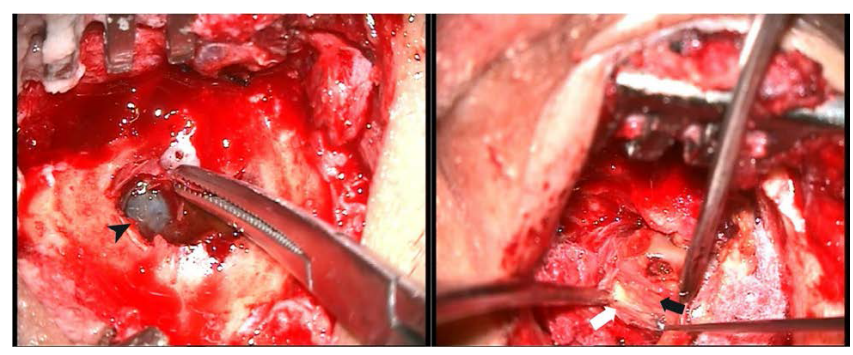

Figure 3: The left photo shows the bony deficits of the sigmoid sinus (arrowhead). The right photo shows the sharp dissector used to separate the thick wall of the cholesterol granuloma (black arrow) from the dura (white arrow).

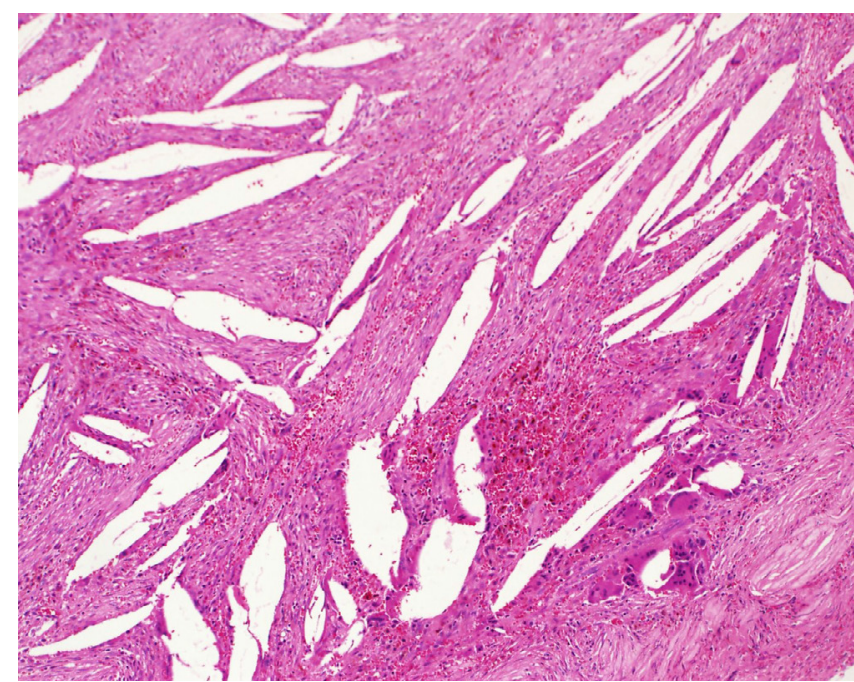

Figure 4: The H\&E stain reveals cholesterol clefts, hemosiderin deposits, and granulomatous inflammation with giant cells. Original magnification, 100x.

proposed: the classic "obstruction-vacuum hypothesis" [4] and the "exposed marrow hypothesis" [5].

Based on the clinical symptoms and radiological features, cholesterol granulomas can be classified as aggressive and nonaggressive [6]. The more common lesions are aggressive cholesterol granulomas that originate from the petrous apex. Non-aggressive cholesterol granulomas usually occur in the tympanomastoid region. Aggressive petrous apex cholesterol granulomas can erode into the cochlea, internal auditory meatus, or middle or posterior cranial fossae, threatening several cranial nerves, including nerves $\mathrm{V}$ and VI.

By contrast, small cholesterol granulomas are usually found accidentally by otologists while performing cholesteatoma surgery or treating chronic otitis media. They are asymptomatic lesions with no involvement of the adjacent structures on imaging and no progression over a long period. They are rarely expansive and destructive of bone. Mastoid cholesterol granulomas are rare, particularly those of an aggressive nature such as that presented here. The preferred treatment is a simple mastoidectomy with insertion of a ventilation tube or with additional mastoid obliteration. In our case, an extended cortical mastoidectomy was performed.

Pfister et al. determined the amount of ongoing hemorrhage required to produce an aggressive cholesterol granuloma in the petrous apex [7]. In our patient, the operative finding suggested that the dura and sigmoid sinus erosion caused continuing hemorrhage, producing an aggressive cholesterol granuloma, which is consistent with Pfister et al. Martin et al. concluded that the difference between a benign and invasive cholesterol granuloma is often self-evident macroscopically [8]: a benign granuloma consists of an agglomeration of cholesterolrich crystal fluid that fills the available potential spaces and is easy to evacuate, whereas the aggressive type is organized into a cyst with a thick wall of inflammatory tissue, and might more properly be labeled a "granuloma". Our case also had a thick wall with bone erosion. Such aggressive true granulomas are very uncommon in the mastoid. The possibility of dural adherence and invasion by this aggressive variant is relevant to the surgical plan. Surgeons must be prepared for dura and sinus repair, and a multidisciplinary approach might be needed.

Our case presented with symptoms often encountered by an 
Citation: Hsieh HS, Zhuo MY, Lee TJ, Zhemg XH, Hwang CF (2014) A Large Mastoid Cholesterol Granuloma Eroding the Dura and Sigmoid Sinus. J Cytol Histol 5: 236. doi:10.4172/2157-7099.1000236

otologist in daily practice. Due to its rarity, however, cholesterol granuloma was not included in the differential diagnosis and its destructive pattern might not be expected preoperatively. We reviewed four other case reports, in which cholesterol granuloma was not included in the differential diagnosis until MRI or surgery was performed [8-11]. The definite diagnosis requires a histological examination. It is crucial to be aware of the invasive nature of these lesions. At otoscopy, the diagnosis of chronic otitis media was made. CT failed to distinguish the cholesterol granuloma from other lesions, such as a cholesteatoma. The MRI characteristics of cholesterol granuloma with high signal intensity on T1- and T2-weighted images facilitates its differentiation from a cholesteatoma, which has low signal intensity on T1-weighted images, but high intensity on T2-weighted images, or from other diseases, and to delineate the anatomic location and involvement of adjacent structures.

\section{Conclusions}

To our knowledge, this is the first report of a cholesterol granuloma that eroded into the cranial cavity and sigmoid sinus. Otologists should be aware of uncharacteristic invasive cholesterol granulomas of the mastoid, which require aggressive surgical obliteration. Preoperative MRI has more diagnostic value than CT in terms of differentiating a cholesterol granuloma from other masses, such as a cholesteatoma.

\section{References}

1. Friedmann I, Graham MD (1979) The ultrastructure of cholesterol granuloma of the middle ear: an electron microscope study. J Laryngol Otol 93: 433-442.
2. Ferlito A (1973) [Cholesteatoma of the middle ear and cholesterol granuloma: histopathological and clinical considerations]. Ann Otolaryngol Chir Cervicofac 90: 697-710.

3. Morais D, Díaz de Tuesta J, Benito JI, Sierra J, Miyar V (1996) [Cholestero granuloma of the temporal lobe]. Acta Otorrinolaringol Esp 47: 317-320.

4. Nager GT, Vanderveen TS (1976) Cholesterol granuloma involving the temporal bone. Ann Otol Rhinol Laryngol 85(2 pt.

5. Jackler RK, Cho M (2003) A new theory to explain the genesis of petrous apex cholesterol granuloma. Otol Neurotol 24: 96-106.

6. Mosnier I, Cyna-Gorse F, Grayeli AB, Fraysse B, Martin C, et al. (2002) Management of cholesterol granulomas of the petrous apex based on clinical and radiologic evaluation. Otol Neurotol 23: 522-528.

7. Pfister $\mathrm{MH}$, Jackler RK, Kunda L (2007) Aggressiveness in cholesterol granuloma of the temporal bone may be determined by the vigor of its blood source. Otol Neurotol 28: 232-235.

8. Martin TP, Tzifa KT, Chavda S, Irving RM (2005) A large and uncharacteristically aggressive cholesterol granuloma of the middle ear. J Laryngol Otol 119: 10011003.

9. Jang $\mathrm{CH}$, Cho YB (2009) Huge cholesterol granuloma of the middle ear extending to middle cranial fossa. In Vivo 23: 191-193.

10. Murugasu E, Yong TT, Yoon CP (2004) Invasive middle ear cholestero granuloma involving the basal turn of the cochlea with profound sensorineural hearing loss. Otol Neurotol 25: 231-235.

11. Masaany M, Siti HS, Nurliza I, Mazita A (2008) Bilateral middle ear cholesterol granuloma in familial hypercholesterolemia. Otolaryngol Head Neck Surg 138 803-804. 